\title{
Analysis of Synchronization Phenomenon in Coupled Oscillator Chains
}

\author{
Kosuke Matsumura, Takahiro Nagai, Yoko Uwate and Yoshifumi Nishio \\ Dept. Electrical and Electronic Eng, Tokushima University, Tokushima 770-8506, JAPAN \\ Email: \{kosuke, nagataka, uwate, nishio\}@ee.tokushima-u.ac.jp
}

\begin{abstract}
In this study, we analyze a synchronization phenomenon observed from a circuit network consisting of several number of oscillator chains which are one-dimensional arrays of weakly coupled van der Pol oscillators. Computer simulations and circuit experiments show interesting unexpected synchronization phenomenon and theoretical analysis explains the synchronization state.
\end{abstract}

\section{INTRODUCTION}

Synchronization phenomena are basic but important phenomena observed everywhere in natures, for example vibration of a pendulum, firefly luminescence, gait patterns of four-leg animals, two frogs using voice religiously, periodic swinging of candle flames, and so on. Coupled oscillators are good models to investigate such interesting synchronization phenomena. Many researchers have proposed different coupled oscillators networks and have discovered many interesting synchronization phenomena [1]-[8]. The research group of the authors is also working on coupled oscillatory networks. Especially, we have been interested in coupled oscillators whose connections cause some kinds of frustrations [9]-[12].

In this study, we consider a circuit network consisting of several number of oscillator chains which are one-dimensional arrays of weakly coupled van der Pol oscillators. In this network, we couple the oscillators at one edge of the chains to constrain them to produce in-phase synchronizations. While we couple the oscillators at the other edge of the chains to produce anti-phase synchronizations. Middle oscillators in the chains are not coupled with the other chains. From computer simulations and circuit experiments, we observe relatively interesting unexpected synchronization phenomenon. By considering the total power consumption of the coupling resistors, we can explain the stable synchronization state theoretically. The results in this study would be a good model of various natural and artificial systems.

\section{Circuit Model}

Figure 1 shows the circuit model for the case of 3 oscillator chains. Each oscillator-chain consists of $n$ van der Pol oscillators weakly coupled by resistors $r$. In the figure, bottom three oscillators are coupled by relatively strong resistors $R_{i}$, while the top three oscillators are coupled by also relatively strong resistors $R_{a}$ via inductors. Middle oscillators are not coupled with oscillators located in the horizontal direction but weakly coupled vertically.
The coupling structure on the bottom or in vertical couplings (by $R_{i}$ or $r$ ) tends to make the oscillators to synchronize in in-phase. While the coupling structure on the top (by $R_{a}$ via inductor) tends to make the oscillators to synchronize in antiphase.

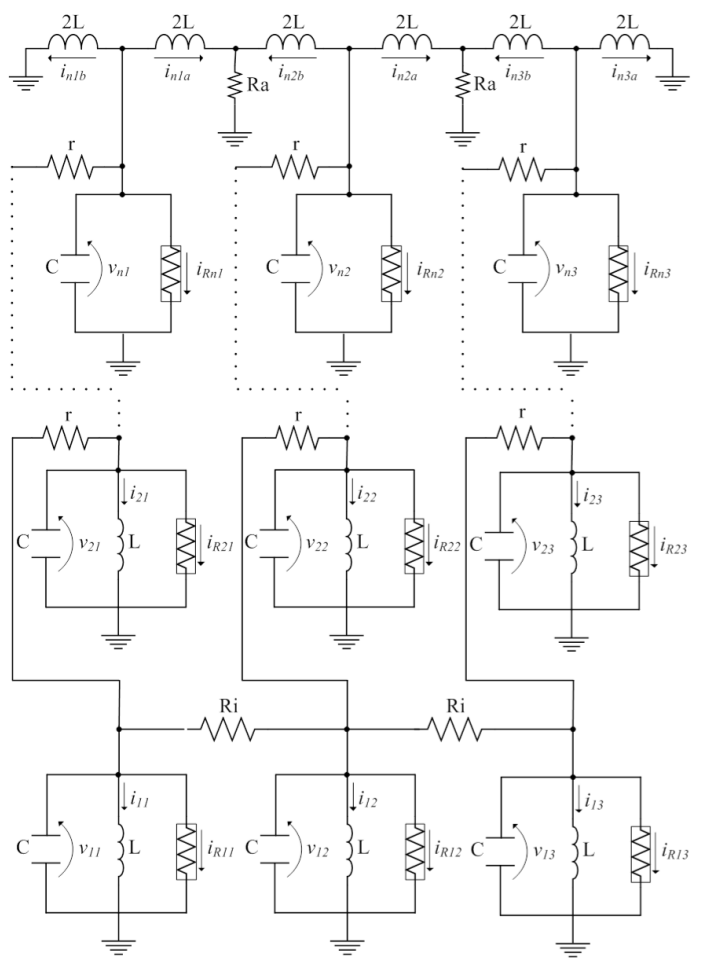

Fig. 1. Circuit model for the case of $\mathrm{n}$ oscillator-chains.

We define the bottom three oscillators as $\mathrm{Osc}_{11}, \mathrm{Osc}_{12}$, and $\mathrm{Osc}_{13}$ from the left, those on the $k$ th row from the bottom as $\mathrm{Osc}_{k 1}, \mathrm{Osc}_{k 2}$, and $\mathrm{Osc}_{k 3}$, and the top three oscillators as Osc $\mathrm{S}_{n 1}$, $\mathrm{Osc}_{n 2}$, and $\mathrm{Osc}_{n 3}$.

First, we assume that the $v-i$ characteristics of the nonlinear resistor in each oscillator is given by the following third order polynomial equation.

$$
i_{R_{k j}}=-g_{1} v_{k j}+g_{3} v_{k j}{ }^{3}
$$

where $g_{1}, g_{3}>0, k=1,2, \ldots, n$, and $j=1,2,3$.

By using the following variables and parameters:

$$
t=\sqrt{L C} \tau, v_{k j}=\sqrt{\frac{g_{1}}{g_{3}}} x_{k j}, i_{k j}=\sqrt{\frac{g_{1} C}{g_{3} L}} y_{k j},
$$




$$
\varepsilon=\frac{1}{g_{1}} \sqrt{\frac{L}{C}}, \alpha_{i}=\frac{1}{R_{i}} \sqrt{\frac{L}{C}}, \alpha_{a}=\frac{1}{R_{a}} \sqrt{\frac{L}{C}}, \beta=\frac{1}{r} \sqrt{\frac{L}{C}},
$$

the normalized circuit equations are given as follows:

(1) Bottom oscillators:

$$
\left\{\begin{aligned}
\dot{x}_{11}=\varepsilon\left(1-x_{11}{ }^{2}\right) x_{11}-y_{11} & +\beta\left(x_{21}-x_{11}\right)-\alpha_{i}\left(x_{11}-x_{12}\right) \\
\dot{y}_{11}=x_{11} & \quad+\alpha_{i}\left(x_{11}-2 x_{12}+x_{13}\right) \\
\dot{x}_{12}=\varepsilon\left(1-x_{12}{ }^{2}\right) x_{12}-y_{12} & +\beta\left(x_{22}-x_{12}\right) \\
\dot{y}_{12}=x_{12} & \\
\dot{x}_{13}=\varepsilon\left(1-x_{13}{ }^{2}\right) x_{13}-y_{13} & +\beta\left(x_{23}-x_{13}\right)-\alpha_{i}\left(x_{13}-x_{12}\right) \\
\dot{y}_{13}=x_{13} &
\end{aligned}\right.
$$

(2) Middle oscillators $(k=2,3, \cdots, n-1)$ :

$$
\left\{\begin{array}{l}
\dot{x}_{k 1}=\varepsilon\left(1-x_{k 1}^{2}\right) x_{k 1}-y_{k 1}+\beta\left(x_{(k+1) 1}-2 x_{k 1}+x_{(k-1) 1}\right) \\
\dot{y}_{k 1}=x_{k 1} \\
\dot{x}_{k 2}=\varepsilon\left(1-x_{k 2}^{2}\right) x_{k 2}-y_{k 2}+\beta\left(x_{(k+1) 2}-2 x_{k 2}+x_{(k-1) 2}\right) \\
\dot{y}_{k 2}=x_{k 2} \\
\dot{x}_{k 3}=\varepsilon\left(1-x_{k 3}^{2}\right) x_{k 3}-y_{k 3}+\beta\left(x_{(k+1) 3}-2 x_{k 3}+x_{(k-1) 3}\right) \\
\dot{y}_{k 3}=x_{k 3}
\end{array}\right.
$$

(3) Top oscillators:

$$
\left\{\begin{array}{l}
\dot{x}_{n 1}=\varepsilon\left(1-x_{n 1}^{2}\right) x_{n 1}-\left(y_{n 1 a}+y_{n 1 b}\right)-\beta\left(x_{n 1}-x_{(n-1) 1}\right) \\
\dot{y}_{n 1 a}=0.5\left\{x_{n 1}-\alpha_{a}\left(y_{n 1 a}+y_{n 2 b}\right)\right\} \\
\dot{y}_{n 1 b}=0.5 x_{n 1} \\
\dot{x}_{n 2}=\varepsilon\left(1-x_{n 2}{ }^{2}\right) x_{n 2}-\left(y_{n 2 a}+y_{n 2 b}\right)-\beta\left(x_{n 2}-x_{(n-1) 2}\right) \\
\dot{y}_{n 2 a}=0.5\left\{x_{n 2}-\alpha_{a}\left(y_{n 2 a}+y_{n 3 b}\right)\right\} \\
\dot{y}_{n 2 b}=0.5\left\{x_{n 2}-\alpha_{a}\left(y_{n 1 a}+y_{n 2 b}\right)\right\} \\
\dot{x}_{n 3}=\varepsilon\left(1-x_{n 3}^{2}\right) x_{n 3}-\left(y_{n 3 a}+y_{n 3 b}\right)-\beta\left(x_{n 3}-x_{(n-1) 3}\right) \\
\dot{y}_{n 3 a}=0.5 x_{n 3} \\
\dot{y}_{n 3 b}=0.5\left\{x_{n 3}-\alpha_{a}\left(y_{n 2 a}+y_{n 3 b}\right)\right\}
\end{array}\right.
$$

where $x_{k j}$ corresponds to the voltage across the capacitor and $y_{k j}, y_{n j a}, y_{n j b}$ are the currents through the inductors of $\mathrm{Osc}_{k j}$.

\section{Synchronization Phenomena}

Figures 2 and 3 show the computer simulation results for the case of $n=3$. The circuit equations (2)-(4) are calculated by using the fourth-order Runge-Kutta method with the step size $h=0.02$. The circuit parameters are chosen as $\varepsilon=0.10$, $\alpha_{i}=\alpha_{a}=0.5$, and $\beta=0.02$.

As we expected, the bottom three oscillators $\left(\operatorname{Osc}_{11}\right.$, Osc $_{12}$, and $\mathrm{Osc}_{13}$ ) are synchronized in in-phase and the top three oscillators $\left(\mathrm{Osc}_{31}, \mathrm{Osc}_{32}\right.$, and $\left.\mathrm{Osc}_{33}\right)$ are synchronized in antiphase.

However, we observe unexpected synchronization phenomenon between the oscillators in each chain. Because the oscillators in each chain are coupled by $r$ vertically, we expected that the three oscillators in the left chain $\left(\mathrm{Osc}_{11}\right.$, $\mathrm{Osc}_{21}$, and $\left.\mathrm{Osc}_{31}\right)$ and in the right chain $\left(\mathrm{Osc}_{13}, \mathrm{Osc}_{23}\right.$, and $\mathrm{Osc}_{33}$ ) are synchronized in in-phase. However, we can observe from Figs. 2 and 3 that the oscillators in the second row $\left(\mathrm{Osc}_{21}\right.$ and $\mathrm{Osc}_{23}$ ) have some amount of phase shift to the first row, and those in the top row $\left(\mathrm{Osc}_{31}\right.$ and $\left.\mathrm{Osc}_{33}\right)$ show larger phase shift.
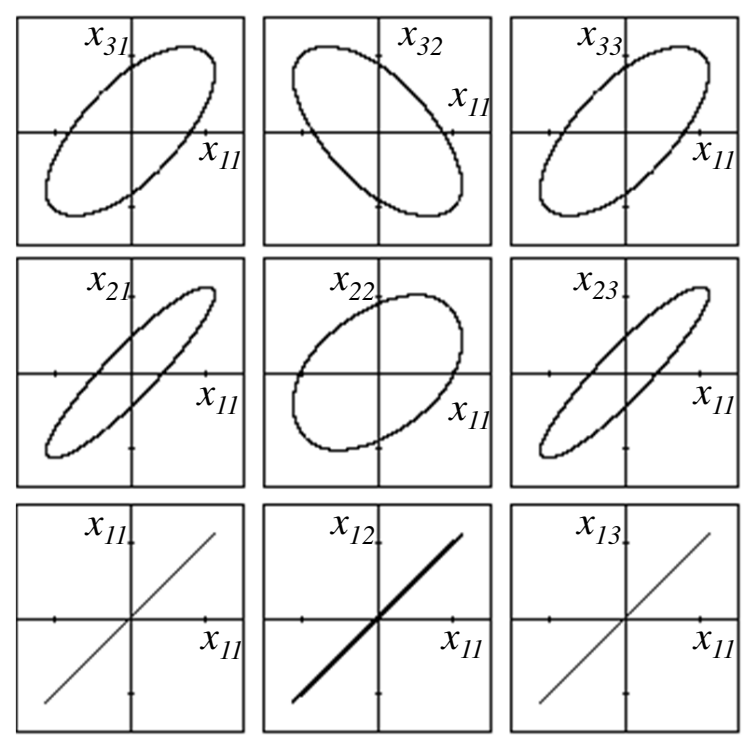

Fig. 2. Computer simulation results (phase shift) for $n=3$.

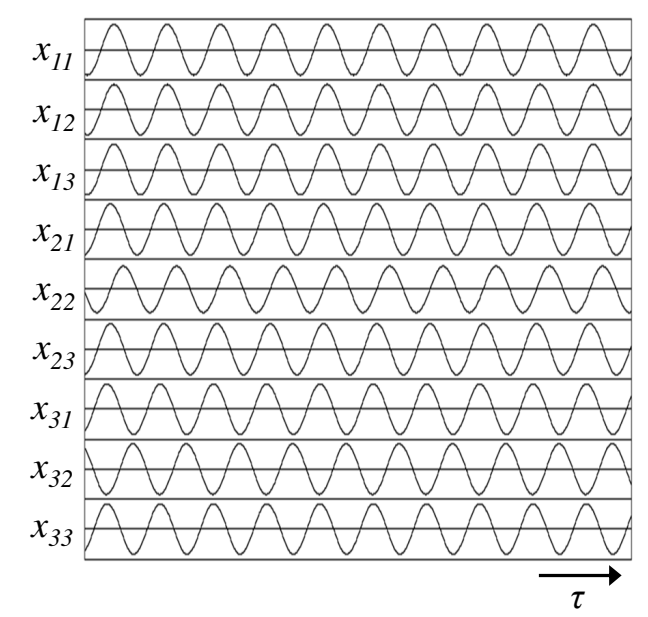

Fig. 3. Computer simulation results (time waveform) for $n=3$.

We could observe the same interesting unexpected synchronization in real circuit experiments. Figure 4 shows the circuit experimental results corresponding to Fig. 2.

\section{Theoretical Analysis}

In order to explain the above-mentioned interesting synchronization phenomenon, we focus on the power consumption of the coupling resistors $r$ in each chain.

First of all, we assume that

1. Oscillators in the bottom and the top rows are synchronized in in-phase and anti-phase, respectively.

2. Phase shift between the neighboring two rows are the same.

These assumptions are reasonable, because of the coupling strength of the bottom and the top rows and the symmetry of the coupling structure.

Under the above assumption, the voltage of each oscillator 

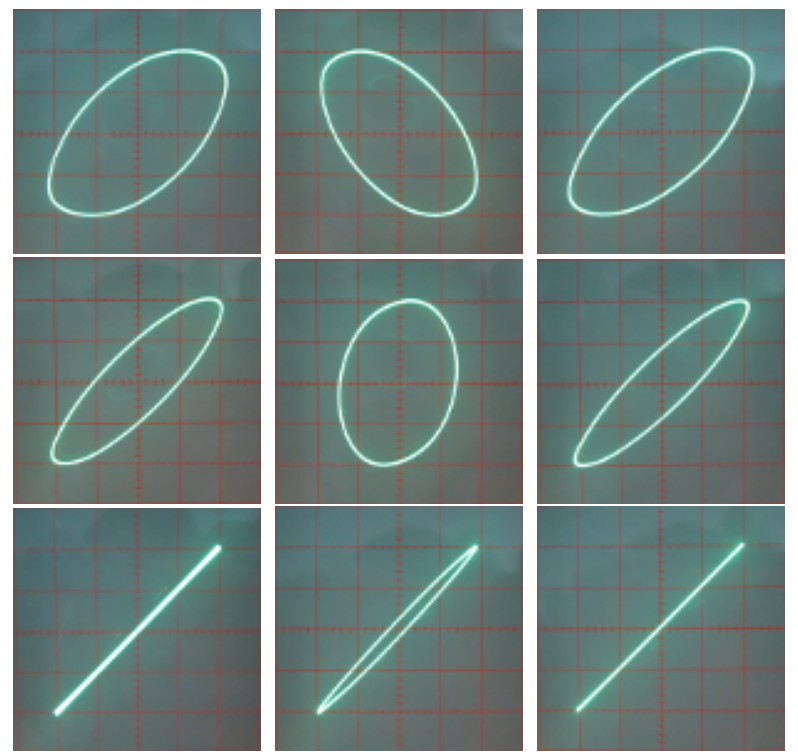

Fig. 4. Circuit experimental results (phase shift) for $n=3$. $C=22 \mathrm{nF}, L=$ $10 \mathrm{mH}, R_{i}=R_{a}=1.0 \mathrm{k} \Omega$, and $r=4.3 \mathrm{k} \Omega$. Horizontal and vertical: $[5.0 \mathrm{~V} / \mathrm{div}]$.

can be written as

$$
\begin{aligned}
& v_{k 1}=v_{k 3}=V_{m} \cos \{\tau+(k-1) \theta\}, \\
& v_{k 2}=V_{m} \cos \left\{\tau+\frac{(k-1)\{(n-1) \theta-\pi\}}{n-1}\right\} .
\end{aligned}
$$

where $\theta$ is a phase shift between two neighboring rows. Also, we define nonlinear oscillators as a first-order approximation.

Next, we consider the power consumption of the resistors in the left and the right chains. Because the phase shift between the two oscillators in these chains is $\theta$, the average power over one period can be calculated as

$$
P_{I}=\frac{1}{r} \int_{0}^{2 \pi}\left(v_{k 1}-v_{(k+1) 1}\right) d \tau=\frac{V^{2}}{r}(1-\cos \theta) .
$$

Next, the power consumption of the resistors in the middle chain can be calculated as follows, because the phase shift between the two oscillators in this chains is $\theta-\pi /(n-1)$.

$$
P_{I I}=\frac{1}{r} \int_{0}^{2 \pi}\left(v_{k 2}-v_{(k+1) 2}\right) d \tau=\frac{V^{2}}{r}\left\{1-\cos \left(\theta-\frac{\pi}{n-1}\right)\right\} .
$$

Hence, the total power consumptions are given as

$$
\begin{aligned}
P_{n} & =2(n-1) P_{I}+(n-1) P_{I I} \\
& =\frac{(n-1) V^{2}}{r}\left\{3-2 \cos \theta-\cos \left(\theta-\frac{\pi}{n-1}\right)\right\} .
\end{aligned}
$$

(A) Case of $n=3$ :

We consider the case of $n=3$. Eq. (8) can be written as

$$
P_{3}=\frac{2 V^{2}}{r}\left\{3-2 \cos \theta-\cos \left(\theta-\frac{\pi}{2}\right)\right\} \text {. }
$$

It is considered that the total power consumption takes the minimum value at the stable synchronization state. In order to find the minimum value of $P_{3}$, we differentiate $P_{3}$ by $\theta$.

$$
\frac{d P_{3}}{d \theta}=\frac{2 V^{2}}{r}(2 \sin \theta-\cos \theta)=0
$$

Calculating Eq. (10), we obtain

$$
\theta=\arctan \frac{1}{2}=26.56[\text { deg. }]
$$

Table I summarizes the phase shifts of all the oscillators obtained by computer simulations and the theoretical analysis. Note that the single value of $\theta$ gives all the phase shifts in theoretical analysis, namely we can calculate all these data from Eq. (5) by substituting the value of Eq. (11). We can see that computer simulation results agree with the data obtained from the theoretical analysis. Small amount of errors are considered to come from the assumptions of the $v-i$ characteristics of the nonlinear resistors Eq. (1) and the constant voltage amplitude Eq. (5).

TABLE I

Phase ShIFTS OF OSCILLATORS FOR $n=3$.

\begin{tabular}{c||c|c||c||c|c}
\hline & $\begin{array}{c}\text { Simulation } \\
{[\mathrm{deg} .]}\end{array}$ & $\begin{array}{c}\text { Theory } \\
{[\mathrm{deg} .]}\end{array}$ & & $\begin{array}{c}\text { Simulation } \\
{[\mathrm{deg} .]}\end{array}$ & $\begin{array}{c}\text { Theory } \\
{[\mathrm{deg} .]}\end{array}$ \\
\hline \hline $\mathrm{Osc}_{11}$ & 0 & 0 & Osc $_{31}$ & 47.31 & 53.13 \\
\hline $\mathrm{Osc}_{12}$ & 0.94 & 0 & $\mathrm{Osc}_{32}$ & 129.13 & 126.87 \\
\hline $\mathrm{Osc}_{13}$ & 0.15 & 0 & $\mathrm{Osc}_{33}$ & 47.31 & 53.13 \\
\hline \hline $\mathrm{Osc}_{21}$ & 23.56 & 26.56 & \multicolumn{4}{|c}{} \\
\hline $\mathrm{Osc}_{22}$ & 64.07 & 63.44 & & & \\
\hline $\mathrm{Osc}_{23}$ & 23.56 & 26.56 & & & \\
\hline
\end{tabular}

(B) Case of $n=9$ :

Next, we consider the case of $n=9$. Computer simulated results are shown in Figs. 5 and 6. We can observe interesting synchronization phenomenon similar to the case of $n=3$, namely the bottom oscillators are synchronized in in-phase and the top oscillators are synchronized in anti-phase, while the middle oscillators (only the oscillators in 3rd, 5th, 7th rows are shown in the figure) look like non-synchronization. However, these phase shifts can be also explained by theoretical analysis by considering the total power consumption.

For the case of $n=9$, Eq. (8) can be written as

$$
P_{9}=\frac{8 V^{2}}{r}\left\{3-2 \cos \theta-\cos \left(\theta-\frac{\pi}{8}\right)\right\} \text {. }
$$

Hence, the stable state can be given by calculating the following equation.

$$
\frac{d P_{9}}{d \theta}=\frac{8 V^{2}}{r}\left\{2 \sin \theta+\sin \left(\theta-\frac{\pi}{8}\right)\right\}=0
$$

Calculating Eq. (13) by the Newton method, we obtain

$$
\theta=7.45[\operatorname{deg} .]
$$

Table II summarizes the phase shifts of some oscillators for the case of $n=9$. The computer simulation results and the theoretical analysis agree even better than the case of $n=3$. 


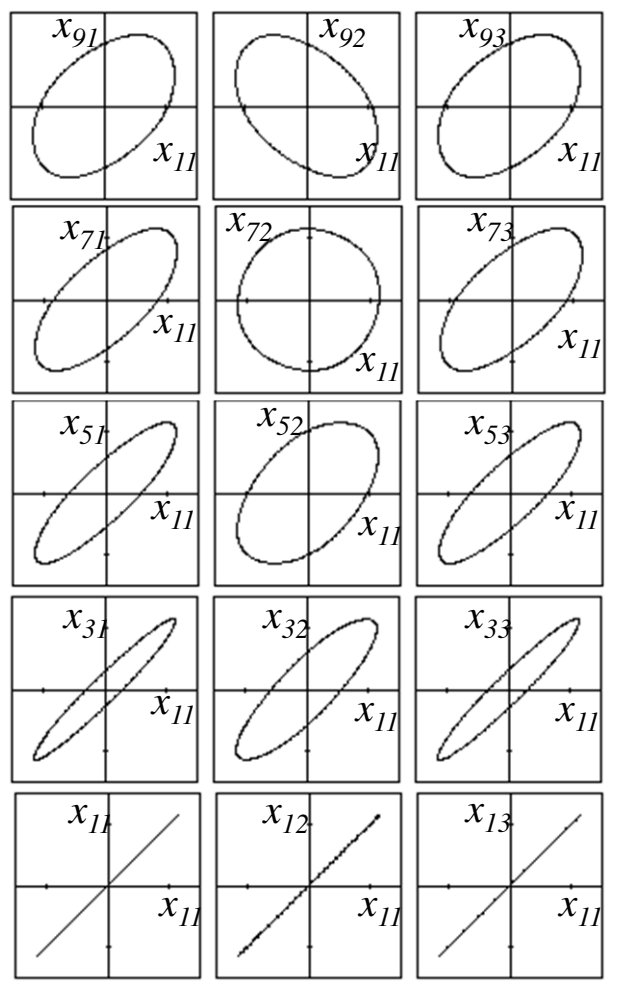

Fig. 5. Computer simulation results (phase shift) for $n=9$.

TABLE II

PHASE SHIFTS OF OSCILLATORS FOR $n=9$.

\begin{tabular}{|c|c|c|c|c|c|}
\hline & $\begin{array}{l}\text { Simulation } \\
\text { [deg.] }\end{array}$ & $\begin{array}{l}\text { Theory } \\
\text { [deg.] }\end{array}$ & & $\begin{array}{l}\text { Simulation } \\
\text { [deg.] }\end{array}$ & $\begin{array}{l}\text { Theory } \\
\text { [deg.] }\end{array}$ \\
\hline Osc $_{11}$ & $\overline{0}$ & $\overline{0}$ & $\mathrm{Osc}_{71}$ & 46.08 & $\overline{44.70}$ \\
\hline Osc $_{12}$ & 0.32 & 0 & $\mathrm{Osc}_{72}$ & 87.37 & 90.30 \\
\hline $\operatorname{Osc}_{13}$ & 0.022 & 0 & $\mathrm{Osc}_{73}$ & 49.98 & 44.70 \\
\hline $\mathrm{Osc}_{31}$ & 14.62 & 14.90 & Osc91 $_{91}$ & 63.12 & 59.60 \\
\hline $\mathrm{Osc}_{32}$ & 30.67 & 30.10 & Osc $_{92}$ & 115.19 & 120.40 \\
\hline $\mathrm{Osc}_{33}$ & 15.90 & 14.90 & $\mathrm{Osc}_{93}$ & 63.85 & 59.60 \\
\hline Osc $_{51}$ & 29.90 & 29.80 & & & \\
\hline $\mathrm{Osc}_{52}$ & 59.56 & 60.20 & & & \\
\hline $\mathrm{Osc}_{53}$ & 32.47 & 29.80 & & & \\
\hline
\end{tabular}

\section{Conclusions}

In this study, we have investigated a synchronization phenomenon observed from coupled oscillator chains whose edges were coupled to constrain their phase states to generate a frustration. By computer simulations and circuit experiments, we could observe interesting unexpected synchronization phenomenon. Theoretical analysis considering the total power consumption of the coupling resistors could explain the stable synchronization state.

The results in this study would be a good model of various natural and artificial systems. For example, stone-paved square of an old town (e.g. we observed one in Evora, Portugal) starts from one edge of the square to line up stones in a regular way like in-phase. However, sometimes the other edge of the square has a different constraint like anti-phase. In that case,

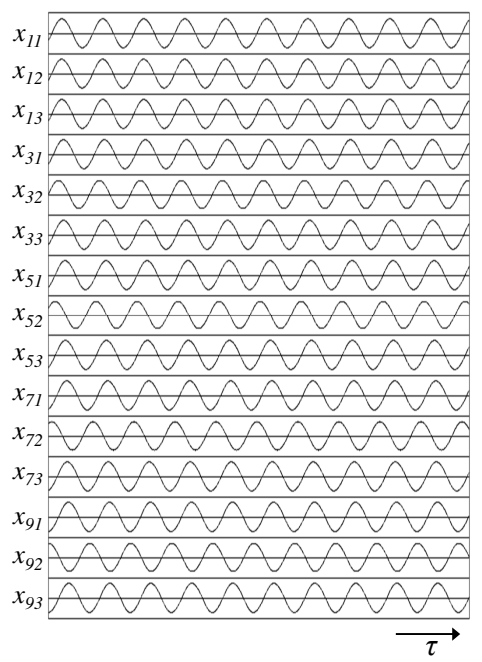

Fig. 6. Computer simulation results (time waveform) for $n=9$.

frustrations occur somewhere in the square and they are not compensated at one or some particular points but over a wide area of the square. That is one example of synchronization phenomena observed from oscillator networks with frustration.

\section{ACKNOWLEDGMENT}

This work was partly supported by JSPS Grant-in-Aid for Young Scientists 23700269.

\section{REFERENCES}

[1] T. Endo and S. Mori, "Mode analysis of a multimode ladder oscillator," IEEE Trans. Circuits Syst., vol. 23, pp. 100-113, Feb. 1976.

[2] T. Endo and S. Mori, "Mode analysis of two-dimensional low-pass multimode oscillator," IEEE Trans. Circuits Syst., vol. 23, pp. 517-530, Sep. 1976.

[3] T. Endo and S. Mori, "Mode analysis of a ring of a large number of mutually coupled van der Pol oscillators," IEEE Trans. Circuits Syst., vol. 25, no. 1, pp. 7-18, Jan. 1978

[4] Y. Nishio and S. Mori, "Mutually coupled oscillators with an extremely large number of steady states," Proc. ISCAS'92, vol. 2, pp. 819-822, May 1992.

[5] S. Moro, Y. Nishio and S. Mori, "Synchronization phenomena in oscillators coupled by one resistor," IEICE Trans. Fundamentals, vol. E78-A, no. 2, pp. 244-253, Feb. 1995.

[6] W. Wei, W. Zhou and T. Chen, "Cluster synchronization of linearly coupled complex networks under pinning control," IEEE Trans. Circuits Syst. I, vol. 56, no. 4, pp. 829-839, Apr. 2009.

[7] Y. Uwate, Y. Nishio and R. Stoop, "Synchronization in three coupled van der Pol oscillators with different coupling strength," Proc. NCSP'10, pp. 109-112, Mar. 2010.

[8] J. Lu, G. Chen and M. Bernardo, "On some recent advances in synchronization and control of complex networks," Proc. ISCAS'10, pp. 37733776, May 2010.

[9] Y. Setou, Y. Nishio and A. Ushida, "Synchronization phenomena in many oscillators coupled by resistors as a ring," Proc. APCCAS'94, pp. 570575, Dec. 1994.

[10] Y. Setou, Y. Nishio and A. Ushida, "Synchronization phenomena in resistively coupled oscillators with different frequencies," IEICE Trans. Fundamentals, vol. E79-A, no. 10, pp. 1575-1580, Oct. 1996.

[11] Y. Uwate, Y. Nishio and R. Stoop, "Synchronization in two polygonal oscillatory networks sharing a branch," Proc. NDES'10, pp. 62-65, May 2010.

[12] Y. Uwate and Y. Nishio, "Synchronizing Coupled Oscillators in Polygonal Networks with Frustration,” Proc. ISCAS'11, pp. 745-748, May 2011. 